\title{
Efficiency Optimization in the Efficiency Similarity Fan Stations
}

\author{
Fulai Yao \\ Department of Mechanical Engineering, \\ University of Connecticut \\ Storrs, Connecticut, USA \\ e-mail: fulai.yao@engineer.uconn.edu
}

\author{
Chengyu Cao \\ Department of Mechanical Engineering, \\ University of Connecticut \\ Storrs, Connecticut, USA \\ e-mail: ccao@uconn.edu
}

\begin{abstract}
This paper defines the normalization efficiency function, the efficiency similarity of different variable-speed fans, and the efficiency similarity fan station. We propose a efficiency optimization method for such fan stations. Based on the efficiency function characteristics which can be approximately considered a concave, non-negative function through the origin, it gives optimal control and optimal switch method and gives the minimum total power consumption. This method is independent of the linearity or nonlinearity of the system and has no need for the system's state equation or transfer function. In the constant output pressure fan station, the optimal control method is to keep the same flow rate for each fan, and the optimal switch points are at the same efficiency points of different operating methods. Since the shapes of efficiency functions in nature are similar, the method given by this paper can be used in other fields.
\end{abstract}

Keywords- variable speed; fan station; efficiency similarity; optimal control; efficiency optimization

\section{INTRODUCTION}

According to statistics, the electricity consumption of fans is $10-15 \%$ of the world's total electricity consumption. With the development of energy-saving, many of speedcontrol equipment such as inverters have been widely applied to the fan stations. A traditional single-close loop control in a variable-speed fan station is shown in Fig .1.

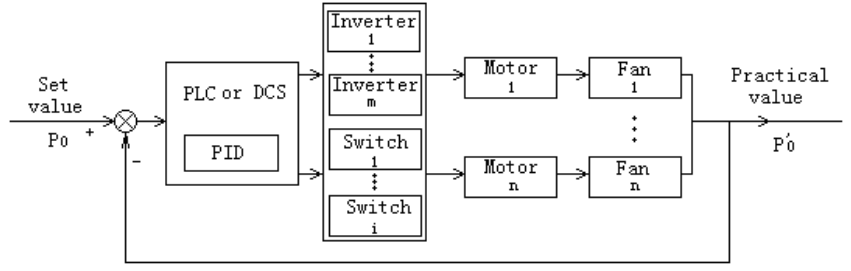

Figure 1. Block diagram of a single-close loop control in fan station

The traditional control is to measure the practical pressure $\mathrm{P}_{0}$ that is to be controlled, compare $\mathrm{P}_{0}$, with the set pressure $\mathrm{P}_{0}$ and feed the error into the PID, the PID changes a manipulated variable to control an inverter that regulates the speed of the motor and fan, and drives $\mathrm{P}_{0}$ back to $\mathrm{P}_{0}$.

However, this control way doesn't consider the total power, so it can not realize the minimum power consumption. With increased awareness for environmental protection and the demand for energy efficient systems, this issue becomes increasingly important. At the present, the studies of optimization in various fan stations are widely studied [1-4]. Almost such optimization approaches need to establish the system's exact model. However, as the exact model of an actual fan station is difficult to establish, these optimization methods can quickly become difficult to apply in an actual system. A scheme that could avoid these complications would be highly advantageous, especially to business and industry.

We can say that, at present, there still haven't optimal control methods which can accurately control energy-saving effect in fan stations [5].

In order to realize the minimum energy, we should decide how many fans are used and how much load each fan undertakes in this fan station.

By supposing the total flow fixed value, we propose an optimal control method akin to conventional static optimization [6], this method does not depend on the exact model of a fan station and is only based on the shape of the efficiency function. By changing the total flow, we also propose a method akin to conventional dynamic optimization [7], this method only depends on the efficiency function of the devices at constant output. These methods can be used for the systems which consist of the same model fans which also have the same efficiency functions.

However, in industrial applications, there still exist a lot of pumping stations in which all fans are not the same model. For solving the optimization of such systems, we expand on the discussion presented in [6,7]. This paper defines a normalized efficiency function $\eta_{N}(Q)$ of a fan and the efficiency similarity fan station consisting of multiple different pumps with the same normalization efficiency function. We are able to find an expression for an arbitrary fan station's power consumption and its minimum value in terms of function $\eta(\beta)$. Our method $[6,7]$ can still be employed when the normalized efficiency functions are the same for each fan. By using the normalized efficiency function determine the minimum power consumption and optimal switch points for such fan station.

We organize the paper in the following manner. Section II gives the motivation and background for the work. In section III we present the problem formulation and the optimization solution. We give a further analysis in section 
IV and apply our results to an optimal switch problem in section $\mathrm{V}$. We give a brief simulation of our work in section VI and conclude the paper in section VII.

\section{MOTIVE AND BACKGROUND}

In general, the efficiency function of a centrifugal variable-speed fan in a output constant pressure $\mathrm{P}_{0}$ fan station has the shape shown in Fig .2.

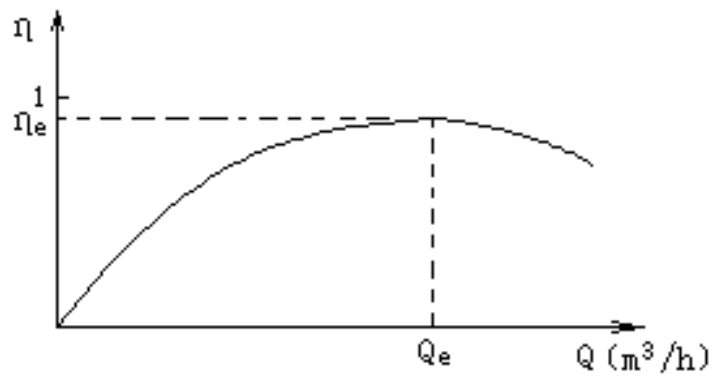

Figure 2. The efficiency function of a centrifugal fan

In Fig .2, $\mathrm{Q}$ is flow ( $\mathrm{m}^{3} /$ hour), and $\eta(\mathrm{Q})$ is the efficiency, $\mathrm{Q}_{\mathrm{e}}$ is the flow at $\eta_{\mathrm{e}}$ which is the maximum efficiency. We have

and

$$
\begin{aligned}
& Q \geq 0 \\
& \eta(Q) \geq 0 \\
& \eta(0)=0
\end{aligned}
$$

$$
\eta^{\prime \prime}(Q)<0
$$

Approximately, $\eta(\mathrm{Q})$ becomes

$$
\eta(Q)=\sum_{i=0}^{\infty} a_{i} Q^{i}=Q \sum_{i=1}^{\infty}\left(a_{i} Q^{i-1}\right) \approx a_{1} Q+a_{2} Q^{2} \geq 0
$$

where

and

$$
\begin{aligned}
& a_{1}+a_{2} Q>0 \\
& a_{1}>0 \\
& a_{2}<0
\end{aligned}
$$

$$
a_{0}=0
$$

The fan's shaft power is N(Q) (kW), it has the form

and

$$
N(Q)=\frac{Q P_{0}}{3600000(Q)}=\frac{N_{0}}{\eta(Q)}
$$

$$
N_{0}=\frac{Q P_{0}}{3600000}
$$

where $\mathrm{N}_{0}$ is ideal work.

If a fan station has n-unit running fans, and the fan station is used to transfer air at the output constant pressure $\mathrm{P}_{0}$, the total flow is $Q_{t}$, all fan's speed is variable, the ith fan's flow is $\mathrm{Q}_{\mathrm{i}}$, then total power consumption has the form

$$
N_{t}\left(Q_{t}\right)=\frac{P_{0}}{3600000} \sum_{i=1}^{n} Q_{i} \frac{1}{\eta_{i}\left(Q_{i}\right)}
$$

and

$$
\sum_{i=1}^{n} Q_{i}=Q_{t}
$$

where $\eta_{i}\left(Q_{i}\right)$ is the ith fan efficiency at the point $\left(Q_{i}, P_{0}\right), N_{t}$ is the total power consumption of the fan station.

The ith fan's power consumption is

$$
N_{i}\left(Q_{i}\right)=\frac{P_{0} Q_{i}}{3600000 \eta_{i}\left(Q_{i}\right)}
$$

If $\mathrm{Q}_{\mathrm{i}}$ equals zero, $\mathrm{N}_{\mathrm{i}}(0)$ may be greater or equal zero. For most real fan station, $N_{i}(0)$ is greater than zero because $\eta_{i}(0)$ equals zero too. In order to avoid the confusion with a stopped fan, we assume $Q_{i}$ is greater than zero for running fan. We then have

$$
Q_{i}>0
$$

\section{THE OPTIMAL CONTROL METHOD OF FAN STATIONS WITH THE SAME FANS[6-7]}

If a fan station has n-unit running fans which are the same model, then all $\eta_{i}\left(Q_{i}\right)$ are the same function $\eta\left(Q_{i}\right)$, and the fan station is used to transfer air at the output constant pressure $\mathrm{P}_{0}$, the ith fan's flow is $\mathrm{Q}_{\mathrm{i}}, \mathrm{Q}_{\mathrm{i}}$ is greater than zero, all fan's speed is variable, the output pressure of the ith fan can be kept a constant value $P_{0}$, the total flow is $Q_{t}$.

We assume $Q_{t}$ is a fixed value. The optimal control method is to keep

$$
Q_{1}=Q_{2}=\ldots=Q_{n}=\frac{Q_{t}}{n}
$$

The minimal value of the total power consumption is

$$
\min N_{t}\left(Q_{t}\right)=N_{0} \frac{1}{\eta\left(\frac{Q_{t}}{n}\right)}
$$

The ideal work $\mathrm{N}_{0}$ is

$$
N_{0}=\frac{Q_{t} P_{0}}{3600000}
$$

The total optimal efficiency is

$$
\max \eta_{t}\left(Q_{t}\right)=\eta\left(\frac{Q_{t}}{n}\right)
$$

\section{THE EFFICIENCY SIMILARITY FAN STATION}

We define the flow rate $\gamma$ as

$$
\gamma=\frac{Q}{Q_{e}}
$$

We also define $\eta_{N}(\gamma)$ to be the normalization efficiency function of a fan. The normalization efficiency function $\eta_{N}(\gamma)$ has a shape shown in Fig .3. 


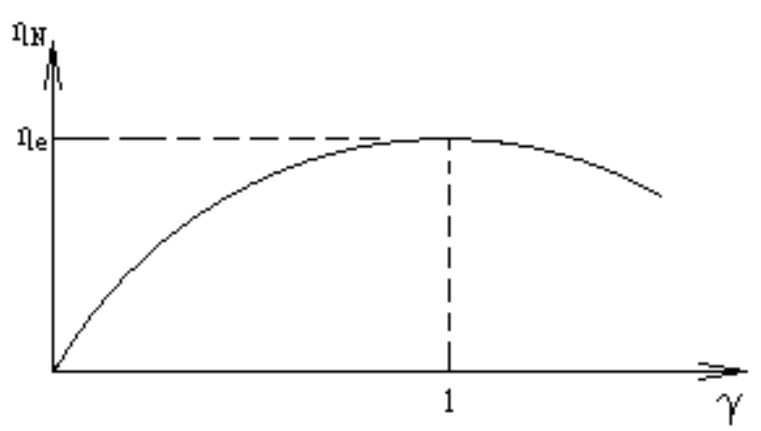

Figure 3. The normalization efficiency function $\eta_{\mathrm{N}}(\gamma)$

In Fig .3, $\gamma$ is a variable and $\eta_{N}(\gamma)$ is the normalized efficiency. $\eta_{\mathrm{N}}(\gamma)$ and $\eta(\mathrm{Q})$ have the relation as following.

$$
\eta(Q)=\eta\left(\gamma Q_{e}\right)=\eta_{N}(\gamma)
$$

If the normalization efficiency functions of two different fans are the same, then

$$
\eta_{N 1}(\gamma)=\eta_{N 2}(\gamma)
$$

We define them as the efficiency similarity fans. The fan station which includes the efficiency similarity fans is called as the efficiency similarity fan station.

Suppose $\gamma_{\mathrm{i}}$ is ith fan's flow rate and has the form

$$
\gamma_{i}=\frac{Q_{i}}{Q_{i e}}
$$

Then for an efficiency similarity fan station, if the fan station is to keep $\mathrm{P}$ as a constant-value $\mathrm{P}_{0}$, the total power consumption has the form

$$
N_{t}\left(Q_{t}\right)=\frac{P_{0}}{3600000} \sum_{i=1}^{n} \frac{Q_{i}}{\eta_{i}\left(Q_{i}\right)}=\frac{P_{0}}{3600000} \sum_{i=1}^{n} \frac{\gamma_{i} Q_{i e}}{\eta_{N}\left(\gamma_{i}\right)}
$$

\section{THE OPTIMAL CONTROL OF EFFICIENCY SIMILARITY FAN STATIONS}

In this section, we use our derived expression for the total power consumption of the efficiency similarity system and establish its minimum power consumption.

Theorem: For the optimization problem of $\mathrm{N}_{\mathrm{t}}\left(\mathrm{Q}_{\mathrm{t}}\right)$, the minimization of the total power consumption

$$
\begin{array}{cc}
\min & N_{t}\left(Q_{t}\right) \\
\text { s.t. } & \gamma_{i}>0, i=1,2, \ldots n \\
& \sum_{i=1}^{n} \gamma_{i} Q_{i e}=Q_{t}
\end{array}
$$

is given by

$$
\min N_{t}\left(Q_{t}\right)=\frac{N_{0}}{\eta_{N}\left(\frac{Q_{t}}{\sum_{i=1}^{n} Q_{i e}}\right)}
$$

Proof: We begin our inductive proof by considering the case where $n=2$. Our equation for the total flow then becomes where

$$
\gamma_{1} Q_{1 e}+\gamma_{2} Q_{2 e}=Q_{t}
$$

$$
\begin{aligned}
& \gamma_{1}>0 \\
& \gamma_{2}>0
\end{aligned}
$$

The objective function $\mathrm{N}_{\mathrm{t}}\left(\mathrm{Q}_{\mathrm{t}}\right)$ is expressed as

$$
N_{t}\left(Q_{t}\right)=\frac{P_{0}}{3600000}\left(\frac{\gamma_{1} Q_{1 e}}{\eta_{N}\left(\gamma_{1}\right)}+\frac{\gamma_{2} Q_{2 e}}{\eta_{N}\left(\gamma_{2}\right)}\right)
$$

The optimal condition is given for

$$
N_{t}^{\prime}\left(\gamma_{1}\right)=0
$$

We have

$$
\frac{Q_{1 e} \eta_{N}\left(\gamma_{1}\right)-\gamma_{1} Q_{1 e} \eta_{N}^{\prime}\left(\gamma_{1}\right)}{\eta_{N}^{2}\left(\gamma_{1}\right)}+\frac{-Q_{1 e} \eta_{N}\left(\frac{Q_{t}-\gamma_{1} Q_{1 e}}{Q_{2 e}}\right)+\left(Q_{t}-\gamma_{1} Q_{1 e}\right) \frac{Q_{1 e}}{Q_{2 e}} \eta_{N}^{\prime}\left(\frac{Q_{t}-\gamma_{1} Q_{1 e}}{Q_{2 e}}\right)}{\eta_{N}^{2}\left(\frac{Q_{t}-\gamma_{1} Q_{1 e}}{Q_{2 e}}\right)}=0
$$

It is then easily verified that

$$
\gamma_{1}=\gamma_{2}=\frac{Q_{t}}{Q_{1 e}+Q_{2 e}}
$$

is a optimal point.

Checking the second derivative, we see

$$
N_{t}^{\prime \prime}\left(\gamma_{1}\right)>0
$$

so the optimal point is only minimum. then

The minimal value of the total power consumption is

$$
\min N_{t}\left(Q_{t}\right)=\frac{P_{0} Q_{t}}{3600000} \frac{1}{\eta_{N}\left(\frac{Q_{t}}{Q_{1 e}+Q_{2 e}}\right)}=\frac{N_{0}}{\eta_{N}\left(\frac{Q_{t}}{Q_{1 e}+Q_{2 e}}\right)}
$$

where $\mathrm{N}_{0}$ is the ideal work.

We then assume that this holds for $n=k$. The above conclusion os readily extended to the case of $n=k$, and the optimal point is then

$$
\gamma_{1}=\gamma_{2}=\ldots=\gamma_{k}=\frac{Q_{t}}{\sum_{i=1}^{k} Q_{i e}}
$$

while the minimal value of the total power consumption is

$$
\min N_{t}\left(Q_{t}\right)=\frac{P_{0} Q_{t}}{3600000} \frac{1}{\eta_{N}\left(\frac{Q_{t}}{\sum_{i=1}^{k} Q_{i e}}\right)}=\frac{N_{0}}{\eta_{N}\left(\frac{Q_{t}}{\sum_{i=1}^{k} Q_{i e}}\right)}
$$

Our inductive case is then given by $n=k+1$. For the total power consumption we have

$$
N_{t}\left(Q_{t}\right)=\frac{P_{0}}{3600000}\left(\sum_{i=1}^{k} \frac{\gamma_{i} Q_{i e}}{\eta_{N}\left(\gamma_{i}\right)}+\frac{\gamma_{k+1} Q_{(k+1) e}}{\eta_{N}\left(\gamma_{k+1}\right)}\right)
$$

and the minimum of the first item is

$$
\min \left(\frac{P_{0}}{3600000} \sum_{i=1}^{k} \frac{\gamma_{i} Q_{i e}}{\eta_{N}\left(\gamma_{i}\right)}\right)=\frac{P_{0}}{3600000} \frac{Q_{t}-\gamma_{k+1} Q_{(k+1) e}}{\eta_{N}\left(\frac{Q_{t}-\gamma_{k+1} Q_{(k+1) e}}{\sum_{i=1}^{k} Q_{i e}}\right)}
$$

where 
and

$$
\gamma_{1}=\gamma_{2}=\ldots=\gamma_{k}
$$

$$
\sum_{i=1}^{k}\left(\gamma_{i} Q_{i e}\right)=\gamma_{1} \sum_{i=1}^{k} Q_{i e}=Q_{t}-\gamma_{k+1} Q_{(k+1) e}
$$

The expression for $\mathrm{N}_{\mathrm{t}}\left(\mathrm{Q}_{\mathrm{t}}\right)$ becomes

$$
N_{t}\left(Q_{t}\right)=\frac{P_{0}}{3600000}\left(\frac{\gamma_{1} \sum_{i=1}^{k} Q_{i e}}{\eta_{N}\left(\gamma_{1}\right)}+\frac{\gamma_{k+1} Q_{(k+1) e}}{\eta_{N}\left(\gamma_{k+1}\right)}\right)
$$

Based on the above conclusion for $n=2$, the optimal point is

$$
\gamma_{1}=\gamma_{k+1}
$$

Therefore the optimal point is then

$$
\gamma_{1}=\gamma_{2}=\ldots=\gamma_{k+1}=\frac{Q_{t}}{\sum_{i=1}^{k+1} Q_{i e}}
$$

and the minimal value of the total power consumption is

$$
\min N_{t}\left(Q_{t}\right)=\frac{P_{0} Q_{t}}{3600000} \frac{1}{\eta_{N}\left(\frac{Q_{t}}{\sum_{i=1}^{k+1} Q_{i e}}\right)}=\frac{N_{0}}{\eta_{N}\left(\frac{Q_{t}}{\sum_{i=1}^{k+1} Q_{i e}}\right)}
$$

Remark: If all of the fans are the same model, the expression will become

$$
\min N_{t}\left(Q_{t}\right)=\frac{N_{0}}{\eta_{N}\left(\frac{Q_{t}}{\sum_{i=1}^{n} Q_{i e}}\right)}=\frac{N_{0}}{\eta\left(\frac{Q_{t}}{n}\right)}
$$

VI. THE OPTIMAL RUNNING NUMBER OF FANS

Suppose a fan station has M-units in total, n-units are running, $n$ is equal or less than $M$. If $n$ is the optimal, there must be

$$
\frac{N_{0}}{\eta_{N}\left(\frac{Q_{t}}{\sum_{i=1}^{n} Q_{i e}}\right)}=\min \left(\frac{N_{0}}{\eta_{N}\left(\frac{Q_{t}}{\sum_{i=1}^{1} Q_{i e}}\right)}, \frac{N_{0}}{\eta_{N}\left(\frac{Q_{t}}{\sum_{i=1}^{2} Q_{i e}}\right)}, \ldots, \frac{N_{0}}{\eta_{N}\left(\frac{Q_{t}}{\sum_{i=1}^{M} Q_{i e}}\right)}\right)
$$

Namely

$$
\eta_{N}\left(\frac{Q_{t}}{\sum_{i=1}^{n} Q_{i e}}\right)=\max \left(\eta_{N}\left(\frac{Q_{t}}{\sum_{i=1}^{2} Q_{i e}}\right), \eta_{N}\left(\frac{Q_{t}}{\sum_{i=1}^{2} Q_{i e}}\right), . ., \eta_{N}\left(\frac{Q_{t}}{\sum_{i=1}^{M} Q_{i e}}\right)\right)
$$

\section{EXTENSION TO THE OPTIMAL SWITCH POINT}

Now we consider the optimal switch point for a efficiency similarity pumping station. Suppose the pumping station has M-units in total. Then we take $n$ to be less than or equal to $\mathrm{M}$ and the optimum. The total optimal efficiency is then

$$
\eta_{N}\left(\frac{Q_{t}}{\sum_{i=1}^{n} Q_{i e}}\right)=\max \left(\eta_{N}\left(\frac{Q_{t}}{\sum_{i=1}^{1} Q_{i e}}\right), \eta_{N}\left(\frac{Q_{t}}{\sum_{i=1}^{2} Q_{i e}}\right), \ldots \eta_{N}\left(\frac{Q_{t}}{\sum_{i=1}^{M} Q_{i e}}\right)\right)
$$

We define $\gamma\left(\mathrm{Q}_{\mathrm{t}}, \mathrm{n}\right)$ as

$$
\gamma\left(Q_{t}, n\right)=\frac{Q_{t}}{\sum_{i=1}^{n} Q_{i e}}
$$

For a variable total flow $Q_{t}$, we see that the optimal switch point is dependent upon whether $Q_{t}$ is increasing or decreasing.

In Fig .4 and Fig .5, the $\eta_{\mathrm{e}}$ is the maximum efficiency, 1 is the flow rate at $\eta_{\mathrm{e}}$. The $\eta_{\mathrm{N}}\left(\mathrm{Q}_{\mathrm{t}}, \mathrm{n}_{1}\right)$ is less than $\eta_{\mathrm{N}}\left(\mathrm{Q}_{\mathrm{t}}, \mathrm{n}\right)$ and greater than other efficiency value on the right side of $\gamma=1$, the $\eta_{N}\left(Q_{t}, n_{2}\right)$ is less than $\eta_{N}\left(Q_{t}, n\right)$ and greater than other efficiency values on the left side of $\gamma=1$.

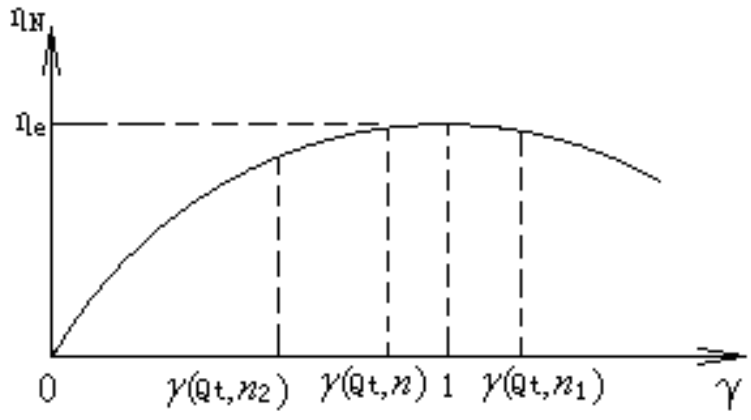

Figue 4. $\eta_{\mathrm{N}}(\gamma)$ curve when $\gamma\left(\mathrm{Q}_{\mathrm{t}}, \mathrm{n}\right)$ is equal or less than 1

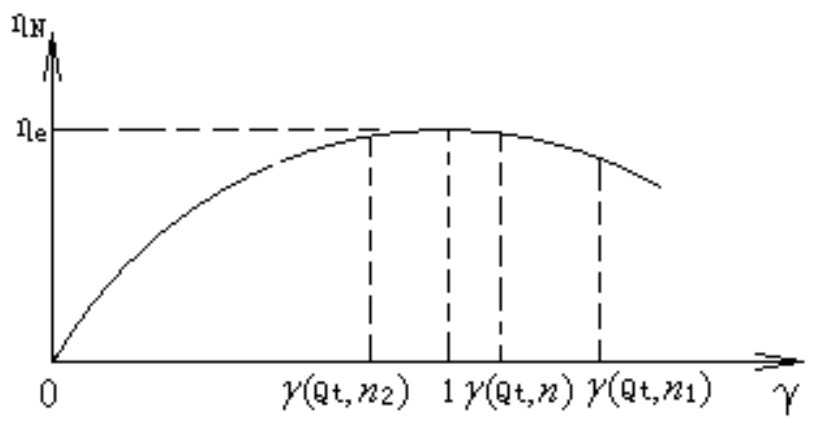

Figure 5. $\eta_{N}(\gamma)$ curve when $\gamma\left(Q_{t}, n\right)$ is greater than 1

Theorem: The optimal switch point for an M-unit system is at $\eta_{N}\left(Q_{t}, n\right)=\eta_{N}\left(Q_{t}, n_{2}\right)$ if $Q_{t}$ is increasing. The optimal switch point for an M-unit system is at $\eta_{N}\left(Q_{t}, n\right)=\eta_{N}\left(Q_{t}\right.$, $\mathrm{n}_{1}$ ) if $\mathrm{Q}_{\mathrm{t}}$ is decreasing.

Proof: From Fig .4 and Fig .5, we see that

$\max \left(\eta_{N}\left(Q_{t}, n_{1}\right), \eta_{N}\left(Q_{t}, n\right), \eta_{N}\left(Q_{t}, n_{2}\right)\right)=\eta_{N}\left(Q_{t}, n\right)$ As seen in Fig .4, if $\gamma\left(Q_{t}, n\right)<1$ and $Q_{t}$ is increases, then $\eta_{N}$ $\left(Q_{t}, n\right)$ will continue to increase until $\gamma\left(Q_{t}, n\right)$ reach 1 , at which point immediately after it will begin to decrease. At the same time, $\eta_{N}\left(Q_{t}, n_{2}\right)$ will increase until it will eventually become greater than $\eta_{N}\left(Q_{t}, n\right)$ and will be the new maximum. This change of the maximum efficiency defines the optimal switch point at $\eta_{N}\left(Q_{t}, n\right)=\eta_{N}\left(Q_{t}, n_{2}\right)$.

If $\gamma\left(Q_{t}, n\right)>1$, then $\eta_{N}\left(Q_{t}, n\right)$ will decrease with the increase of $Q_{t}$ while $\eta_{N}\left(Q_{t}, n_{2}\right)$ will increase as can be 
inferred from Fig .5. Ultimately, $\eta_{N}\left(Q_{t}, n_{2}\right)$ will increase to such a point that it will become the new maximum and $\eta_{\mathrm{N}}$ $\left(Q_{t}, n\right)=\eta_{N}\left(Q_{t}, n_{2}\right)$ is the optimal switch point.

In a manner akin to our arguments for an increasing $Q_{t}$, we begin by looking at $\gamma\left(\mathrm{Q}_{\mathrm{t}}, \mathrm{n}\right)>1$. If $\mathrm{Q}_{\mathrm{t}}$ is decreasing, then Fig .5 shows that $\eta_{N}\left(Q_{t}, n\right)$ will increase until $\gamma\left(Q_{t}, n\right)$ reaches the value of 1 , where it will immediately begin to decrease. Simultaneously, $\eta_{N}\left(Q_{t}, n_{1}\right)$ will increase. As before, there will be point in which $\eta_{N}\left(Q_{t}, n_{1}\right)$ overtakes $\eta_{N}$ $\left(Q_{t}, n\right)$ as the new maximum. This is our optimal switch point and is given by $\eta_{N}\left(Q_{t}, n\right)=\eta_{N}\left(Q_{t}, n_{1}\right)$.

As seen in Fig .3, for a given $\gamma\left(Q_{t}, n\right)<1, \eta_{N}\left(Q_{t}, n\right)$ will decrease as $Q_{t}$ decreases. We also have that $\eta_{N}\left(Q_{t}, n_{1}\right)$ will increase to the point where it will be the maximum efficiency. The optimal switch point is then $\eta_{N}\left(Q_{t}, n\right)=\eta_{N}\left(Q_{t}, n_{1}\right)$.

\section{SIMULATION}

If a fan station is used to transfer air at the output constant pressure $0.6 \mathrm{Mpa}$, and the station consists of two different fans, A and B, each with adjustable speed.

Fan $\mathrm{A}$ is smaller and its efficiency function about the flow $\mathrm{Q}$ with its out at $0.6 \mathrm{Mpa}$ is given by

where

$$
\eta_{1}(Q)=0.0168 Q-0.000084 Q^{2}
$$

$$
Q_{1 e}=100\left(m^{3} / h\right)
$$

and

$$
\eta_{1 e}=0.84
$$

Fan B is larger, and its efficiency function about the flow $\mathrm{Q}$ at $0.6 \mathrm{Mpa}$ is given by

$$
\eta_{2}(Q)=0.0084 Q-0.00002 Q^{2}
$$

where

$$
Q_{2 e}=200\left(m^{3} / h\right)
$$

and

$$
\eta_{2 e}=0.84
$$

We see that $\mathrm{A}$ and $\mathrm{B}$ have the same normalization efficiency function, given by the following

$$
\eta_{N}(\gamma)=1.68 \gamma-0.84 \gamma^{2}
$$

Based on our previous conclusions, the optimal switch point between $\mathrm{A}$ and $\mathrm{B}$ is at

$$
1.68\left(\frac{Q_{t}}{Q_{1 e}}\right)-0.84\left(\frac{Q_{t}}{Q_{1 e}}\right)^{2}=1.68\left(\frac{Q_{t}}{Q_{2 e}}\right)-0.84\left(\frac{Q_{t}}{Q_{2 e}}\right)^{2}
$$

Solving this equation for $Q_{t}$, we see that the optimal switch point between pumps $\mathrm{A}$ and $\mathrm{B}$ is

$$
Q_{t}=133.33=Q_{A-B}
$$

Thus, when the total flow $Q_{t}$ is less than $Q_{A-B}$, only fan $A$ should run, and when $Q_{t}$ is greater than $Q_{A-B}$ only fan $B$ should run. Therefore, $\mathrm{Q}_{\mathrm{A}-\mathrm{B}}$ is the optimal switch point.

Similarly, we can extend this and determine when the system should switch from the large fan B and run both fans simultaneously. The optimal switch point between $B$ and $\mathrm{A}+\mathrm{B}$ is found by solving the following for $\mathrm{Q}_{t}$
$1.68\left(\frac{Q_{t}}{Q_{2 e}}\right)-0.84\left(\frac{Q_{t}}{Q_{2 e}}\right)^{2}=1.68\left(\frac{Q_{t}}{Q_{1 e}+Q_{2 e}}\right)-0.84\left(\frac{Q_{t}}{Q_{1 e}+Q_{2 e}}\right)^{2}$

Our optimal switch point between $\mathrm{B}$ and $\mathrm{A}+\mathrm{B}$ is then

$$
Q_{t}=240=Q_{B-(A+B)} \text {. }
$$

We see that when the total flow $\mathrm{Q}_{t}$ is less than $\mathrm{Q}_{\mathrm{B}-(\mathrm{A}+\mathrm{B})}$ and greater than $\mathrm{Q}_{\mathrm{A}-\mathrm{B}}$, only fan $\mathrm{B}$ should run. When $\mathrm{Q}_{t}$ becomes

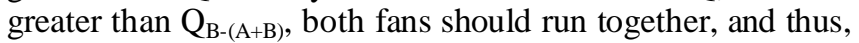
$\mathrm{Q}_{\mathrm{B}-(\mathrm{A}+\mathrm{B})}$ is the optimal switch point. The optimal control method is to have fan A and B keep the same flow rate

$$
\gamma_{1}=\gamma_{2}=\frac{Q_{t}}{Q_{1 e}+Q_{2 e}}=\frac{Q_{t}}{300} \text {. }
$$

\section{THE CONCLUSION}

The Proof of the optimal control and switch method is given by this paper is mainly based on the efficiency function characteristics which can be approximately considered a concave, non-negative function through the origin. Thusly, the optimal method is independent of the linearity or nonlinearity of the system and has no need for the system's state equation or transfer function. Since the shapes of efficiency functions in nature are similar, the method given by this paper can be used in other fields. To resolve the optimization problems of general devices, the authors have done extensive research [8-11].

\section{REFERENCES}

[1] Fourie, J.W. Power savings by impeller replacements for main fan stations in the South African gold mining industry, Industrial and Commercial Use of Energy Conference, 2012 Proceedings of the 9th, Page(s): 1-6,2012

[2] Oliver, J.A. ; Weiss, H.W. ; McCluskey, R.K. ; Samotyj, M.J. Adjustable-speed drive retrofit for Ormond Beach FD fans, IEEE Transactions on Energy Conversion, Volume: 7, Issue: 3, Page(s): $580-588,1992$

[3] Matsui, N. ; Kurokawa, F. Improvement of Transient Response of Thermal Power Plant Using VVVF Inverter. 7th International Conference on Power Electronics and Drive Systems, Page(s): 12091214,2007

[4] Bhowmick, M.S. ; Bera, S.C. Study the Performances of Induced Fans and Design of New Induced Fan for the Efficiency Improvement of a Thermal Power Plant, IEEE Region 10 and the Third international Conference on Industrial and Information Systems, Page(s): 1-5, 2008

[5] Fulai Yao And Hexu Sun, Efficiency optimal control and dispatching method for General equipment, 2012, China Machine Press, china

[6] Fulai Yao And Hexu Sun, Optimal Control in Variable-speed Fan Stations, IEEE The 18th International Conference on Industrial Engineering and Engineering Management, September 3-5,2011. China.

[7] Fulai Yao And Hexu Sun, Optimal Switch in Variable-speed Fan Stations, 2011 International Conference of Renewable Energy Sources and Environmental Materials, May 20-22, 2011, China.

[8] Fulai Yao And Hexu Sun, Optimal Control in Variable-speed Pumping Stations, The 2011 IEEE International Conference on Mechatronics and Automation, August 7-10, 2011,Beijing, China.

[9] Fulai Yao, Optimal Switch in Variable-speed Pumping Stations;2011 IEEE 2nd International Conference on computing, control and Industrial Engineering, August 20-21, 2011, China. 
[10] Fulai Yao And Hexu Sun, Optimal Control for a Common System, 2011 3rd IEEE International Conference on Information management and engineering, May 21-22, 2011, Zhengzhou, China
[11] Fulai Yao And Hexu Sun, Optimal Switch for a Common System, IEEE Power Engineering and Automation Conference, September 89, 2011 in Wuhan, China 